\title{
Analysis of the operation of PV strings at the MPP closest to the nominal MPP voltage instead of the global MPP based on measured current-voltage curves
}

\author{
Kari Lappalainen ${ }^{*}$ (D) and Seppo Valkealahti \\ Tampere University, Electrical Engineering Unit, P.O. Box 692, 33101 Tampere, Finland
}

Received: 28 June 2021 / Received in final form: 29 November 2021 / Accepted: 3 January 2022

\begin{abstract}
Under non-uniform operating conditions, photovoltaic (PV) generators may have several maximum power points (MPP) and voltage of the global MPP (GMPP) may vary quickly over a wide voltage range which may cause problems for tracking of the GMPP. Since highly varying GMPP voltage causes fluctuation of the inverter reference voltage, it would be beneficial to operate the PV system in a more predictable and straightforward manner by keeping the operating point of the inverter all the time close to the nominal MPP voltage. This article presents an experimental study of a scenario in which the MPP closest to the nominal MPP voltage (CMPP) is always the operating point instead of the GMPP. The analysis was based on 1,296,000 measured current-voltage curves of three different PV strings located at Tampere, Finland. 12 days of full-time measurements were analysed for each of the studied strings consisting of 6,17 and 23 series-connected NAPS NP190GK PV modules. Furthermore, the effects of inverter sizing on the operating point behaviour of the strings were studied. The results show that the wide operating voltage range of the GMPP can be significantly reduced by operating at the CMPP at a cost of negligible energy losses. Energy losses due to power curtailment were much larger than energy losses due to operation at the CMPP instead of the GMPP.
\end{abstract}

Keywords: Photovoltaic power generation / maximum power point / operating voltage / inverter sizing / power variation

\section{Introduction}

Photovoltaic $(\mathrm{PV})$ power generators are constantly prone to variation in their operating conditions. Especially, fast irradiance fluctuations due to overpassing cloud shadows can have negative effects on the operation of PV generators and electrical grids causing fast fluctuations in the power fed to the grid. In order to secure undisturbed operation of the grids, some transmission system operators have already restricted the allowed power variations of grid-connected PV power plants [1]. There are two typical ways to restrict the output power of PV power plants to comply with power variation requirements: power output curtailment and energy storage systems [2]. However, the power output curtailment can be used to restrict only upward power ramps [3].

During homogeneous operating conditions, the electrical characteristic of a PV generator has exactly one peak, i.e., maximum power point (MPP). However, during nonuniform operating conditions, the PV cells of the generator have divergent electrical characteristics, and as a result,

\footnotetext{
* e-mail: kari.lappalainen@tuni.fi
}

the electrical characteristic of the whole generator may have several MPPs. Only one of the MPPs represents true maximum power. This point is called the global MPP (GMPP) while the other MPPs are called local MPPs (LMPP). A typical reason for non-uniform operating conditions of the generator is partial shading. In addition to the existence of multiple MPPs, partial shading can cause mismatch losses $[4,5]$. The electrical and thermal characteristics of partially shaded PV arrays were analysed in [6].

Existence of multiple MPPs complicates MPP tracking (MPPT) and can lead to operation at an LMPP instead of the GMPP. Thus, several new MPPT algorithms for tracking the GMPP have been developed over the past few years to extract the highest possible output power under the existence of multiple MPPs [7,8]. Moreover, the voltage of the GMPP can vary quickly over a wide voltage range [9]. Multiple MPPs and fast fluctuations in the GMPP voltage mostly exist only under extensive irradiance variation over the PV generator. In well-designed PV power plants, the only source of extensive irradiance variations is shading by clouds. Most inverters have a certain allowed voltage range for proper operation and, accordingly, applied MPPT algorithms have defined 
operational voltage ranges to ensure that the GMPP is followed under changing operating conditions. Thus, the knowledge of the applicable operating range of the GMPP voltage of the installed PV generator is important for proper selection of the inverter voltage range.

$\mathrm{PV}$ generators are typically oversized with respect to their inverters such that the generator nominal DC power is higher than the inverter nominal AC power [10]. Oversizing of PV capacity limits the output power of the $\mathrm{PV}$ generator to the inverter nominal power during high irradiance conditions. If the GMPP power of the generator is higher than the inverter maximum power, the inverter will operate in power limiting mode meaning that the operating point of the inverter is moved to higher voltages to decrease the current and power of the inverter. In power limiting mode, variations in DC power are not transmitted to $\mathrm{AC}$ power. Operating in power limiting mode causes energy losses compared to operation at the GMPP. Moreover, high operating voltages affect the operation and efficiency of the inverter: the inverter capacitor lifetime shortens [11] and the efficiency of some inverters decreases [12] with increasing DC side voltage. The optimal sizing of the inverter depends on many factors such as inverter characteristics and irradiance conditions [13]. The authors of [14] recommended that $\mathrm{DC} / \mathrm{AC}$ ratio, i.e., the ratio of the nominal DC power of the PV string to the inverter nominal AC power, should be from 1.1 to 1.7 . It was found in $[15,16]$ that $\mathrm{DC} / \mathrm{AC}$ ratio should be less than 1.0 if the intention is to avoid all power curtailment. In accord with [17], region of the optimal $\mathrm{DC} / \mathrm{AC}$ ratio is fairly flat and up to $20 \%$ changes from the optimal DC/AC ratio usually leads to less than $2 \%$ energy losses. Inverter sizing has an enormous impact on the operating point of the PV generator. However, the effects of inverter sizing on the optimal operating point of $\mathrm{PV}$ generators have not been studied comprehensively.

Although a lot of research and development work is going on to improve PV system efficiencies and several novel MPPT algorithms have been proposed over the past few years, the actual MPP characteristics of PV generators have received little attention. MPP characteristics of PV generators have been studied by simulations in [18-22] and based on electrical measurements in [9,23]. However, fictitious irradiance values were used in $[18,21,22]$ and only the partially shaded time of the studied strings was considered in $[9,23]$. Furthermore, the effects of inverter sizing on the operating point of $\mathrm{PV}$ generators were not considered in these studies. In [18,22], the highest GMPP voltage of a string of series-connected PV modules was found to be under $90 \%$ of the nominal open-circuit (OC) voltage while the lowest GMPP voltage can be very low. In [20], these results were confirmed also for larger PV arrays consisting of multiple parallel PV strings. The main reason why earlier studies related to MPP characteristics of PV generators have typically been based on simulations instead of actual measurements is that PV string or array level current-voltage $(I-U)$ curve measurements are not available from many places. In this study, the shortcomings of earlier studies of this topical area are eliminated by analysing an extremely extensive set of measured $I-U$ curves of 3 different PV strings also considering the effects of inverter sizing on the operating point.

Since highly varying GMPP voltage causes large fluctuation of the inverter reference voltage, posing challenges for MPPT, it would be beneficial to keep the operating point of the inverter all the time at voltages close to the nominal MPP voltage. In this way, the operation of the PV system would be smoother, more straightforward and more predictable. In this article, an experimental study is presented of the scenario in which the MPP closest to the nominal MPP voltage (CMPP) is always the operating point instead of the GMPP. The study is based on measured $I-U$ curves of $3 \mathrm{PV}$ strings located at Tampere, Finland. In total, almost 1.3 million $I-U$ curves measured over $360 \mathrm{~h}$ are analysed. Furthermore, the effects of inverter sizing on the operating point behaviour of the $\mathrm{PV}$ strings are studied. The main novelty of the study is that, for the first time, the MPP behaviour of PV strings is analysed comprehensively based on an extensive set of actual full-time electrical measurements. Such an exhaustive study based on actual electrical measurements of the optimal operating point of PV generators, also considering the effects of inverter sizing on the selection of the operating point, has not been presented earlier. The results of this study are particularly relevant for PV power plant design and for development of MPPT algorithms while trying to achieve higher overall efficiency and power quality of PV systems. The rest of this article is organised as follows. The used measurement setup, experimental data and $I-U$ curve pre-processing procedure are introduced in Section 2. Section 3 presents the obtained experimental results and their analysis. Further discussion on the results and their significance is given in Section 4. Finally, conclusions of the article are presented in Section 5.

\section{Experimental data and methods}

In this study, almost 1.3 million $I-U$ curves measured over $360 \mathrm{~h}$. were utilised to research the MPP behaviour of PV strings. 12 days of full-time measurements of $3 \mathrm{PV}$ strings were analysed. The analysed measurements of each string were performed during 2 periods of 6 consecutive days. Measurement period of each day was from 8:00 to 18:00 $(\mathrm{UTC}+2)$. Partial layout scheme of the PV power research plant of Tampere University [24] showing the studied strings is presented in Figure 1. Strings 1 and 4 consist of 17 and 6 series-connected PV modules, respectively. Moreover, the series connection of Strings 1 and 4, consisting of $23 \mathrm{PV}$ modules, was studied. The series connection of Strings 1 and 4 is referred to as String $1 \& 4$. The studied PV strings consist of NAPS NP190GK modules, which are composed of 3 submodules of 18 polycrystalline silicon solar cells. Each submodule is protected by an anti-parallelconnected bypass diode. The standard test conditions (STC) power $(P)$, voltage and current values of the PV modules are compiled in Table 1 and the details of the studied strings are compiled in Table 2. Seven modules of 


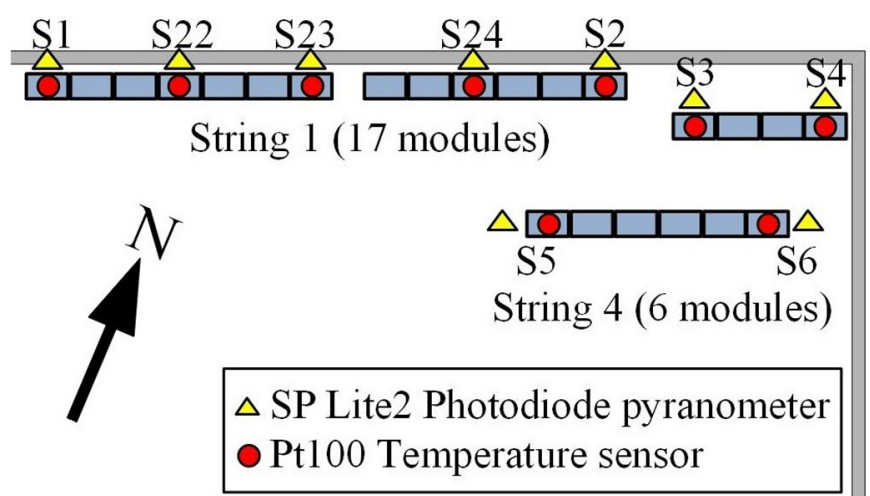

Fig. 1. Partial layout scheme of the PV power research plant of Tampere University indicating the locations of the PV modules, irradiance sensors and temperature sensors of the studied PV strings.

String 1 and two modules of String 4 are equipped with irradiance and temperature measurements with a sampling frequency of $10 \mathrm{~Hz}$. Irradiance incident on the modules was measured by SP Lite2 pyranometers mounted at the same $157^{\circ}$ azimuth angle from north to east and $45^{\circ}$ tilt angle as the PV modules. Back-sheet temperature of the PV modules was captured by Pt100 temperature sensors. Figure 2 further illustrates the studied strings and the locations of the pyranometers. The pyranometers of String 4 are installed on the bottom part of the modules side while the rest of the pyranometers are installed on the top edge of the modules. The modules are installed on the rooftop of a campus building at the same azimuth angle with the building at nearly optimal tilt angle.

An $I-U$ curve was measured once a second during the measurement period of $360 \mathrm{~h}$. Thus, the total number of analysed measured $I-U$ curves is $1,296,000$. The $I-U$ curves were traced using an $I-U$ curve tracer based on the electronic load method by loading the PV string with a dynamic resistance that can alter the output current of the string. Parallel-connected IGBTs act as an electronic load and are gate controlled with a ramp signal for opening and closing channels of the transistors. The voltage was measured by LeCroy AP031 differential voltage probe and the current by Tektronix TCP312A current probe with Tektronix TCPA300 current probe amplifier. The measurement sweep direction of the tracer is from OC to SC, and each measured $I-U$ curve involves 4000 measurement points.

The measured $I-U$ curves were pre-processed by the following procedure. First, the measurement points with identical voltage value were replaced with a single point by averaging their current values. Thereafter, clearly abnormal measurement points were removed. A point was considered abnormal and removed if its power differed from the power of the previous and next point (to the same direction) by more than 1.3 times the mean change of power between adjacent measurement points in its vicinity (previous and next 9 points). Lastly, the measured current and voltage were smoothed separately using smooth function in MATLAB. An example of an original and
Table 1. STC parameter values of NAPS NP190GK PV modules.

\begin{tabular}{ll}
\hline Parameter & Value \\
\hline$P_{\text {MPP, STC }}$ & $190 \mathrm{~W}$ \\
$U_{\text {MPP, STC }}$ & $25.8 \mathrm{~V}$ \\
$I_{\text {MPP, STC }}$ & $7.36 \mathrm{~A}$ \\
$U_{\text {OC, STC }}$ & $33.0 \mathrm{~V}$ \\
\hline
\end{tabular}

pre-processed measured $P-U$ curve of String $1 \& 4$ is shown in Figure 3 that illustrates the pre-processing method.

The analysed $I-U$ curves were measured in August 2020 and April-June 2021. The analysed measurements of String 1 were performed on 14-19 August 2020 and 23-28 April 2021, the measurements of String 4 on 7-12 August 2020 and 14-19 May 2021, and the measurements of String $1 \& 4$ on $7-12$ May and 3-8 June 2021. Figure 4 shows the distributions of the average irradiances of the studied PV strings. The distributions of the strings are quite similar, indicating that there were no major differences in the irradiance conditions during the measurement periods of the strings. Each distribution has two peaks: higher around $100 \mathrm{~W} / \mathrm{m}^{2}$ and lower around $900 \mathrm{~W} / \mathrm{m}^{2}$ associated with cloudy periods with only diffuse radiation and clear sky sunny periods, respectively. The highest measured average irradiances were 1413, 1195 and $1302 \mathrm{~W} / \mathrm{m}^{2}$, for Strings 1, 4 and $1 \& 4$, respectively. These very high irradiance readings are caused by a phenomenon called irradiance enhancement or cloud enhancement [15]. Irradiance under partly cloudy conditions can be higher than under clear sky since photons scattering off clouds near the direct path of sunbeams. In the Tampere region, the highest theoretical clear sky irradiance for PV modules installed at an optimal angle is just over $900 \mathrm{~W} / \mathrm{m}^{2}$.

Shares of time when the studied PV strings were partially shaded, i.e., the difference between the lowest and highest irradiance measurement of the string exceeded a certain limit are compiled in Table 3. Irradiance differences of over $50 \mathrm{~W} / \mathrm{m}^{2}$ were common for Strings 1 and $1 \& 4$. Partial shading conditions were much rarer for the physically shortest String 4, which is an expected result. However, it is worth noting that String 4 is equipped with only two irradiance sensors and the distance between the sensors is longer than in String 1 (see Fig. 1). Thus, there might have been some shadings between the irradiance sensors of String 4 that are not included in the values of Table 3. However, that kind of shadings are not probable since typical sizes of cloud shadows are several hundred meters [25]. Naturally, the share of time of partial shading decreased with increasing irradiance difference limit and irradiance differences of over $200 \mathrm{~W} / \mathrm{m}^{2}$ were rare for all the studied strings.

The effects of inverter sizing on the MPP behaviour of the PV strings were studied by altering the $\mathrm{DC} / \mathrm{AC}$ ratio from 0.8 to 2.0. The lower limit was selected based on the highest measured average irradiances of the studied strings. The $\mathrm{DC} / \mathrm{AC}$ ratio of 0.8 means that the inverter nominal power is 1.25 times the nominal string power. 
Table 2. Details of the studied PV strings. The nominal STC values were calculated using the STC values of NAPS NP190GK PV modules (see Tab. 1).

\begin{tabular}{lllll}
\hline String & Number of modules & $U_{\mathrm{MPP}, \mathrm{STC}}(\mathrm{V})$ & $P_{\mathrm{MPP}, \mathrm{STC}}(\mathrm{W})$ & $U_{\mathrm{OC}, \mathrm{STC}}(\mathrm{V})$ \\
\hline 1 & 17 & 439 & 3230 & 561 \\
4 & 6 & 155 & 1140 & 198 \\
$1 \& 4$ & 23 & 593 & 4370 & 759 \\
\hline
\end{tabular}

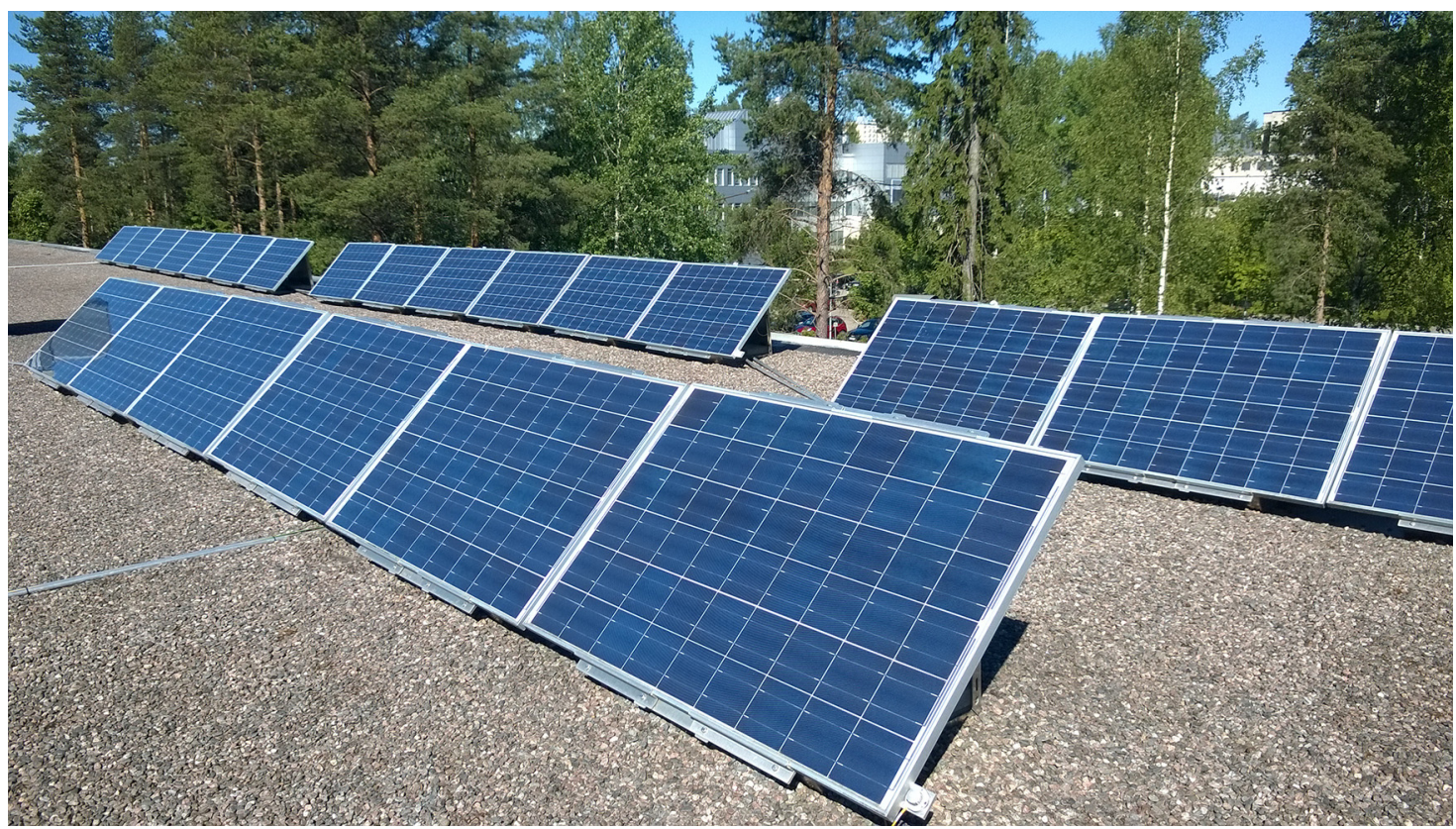

Fig. 2. Photograph of the studied PV strings on the rooftop of a campus building.

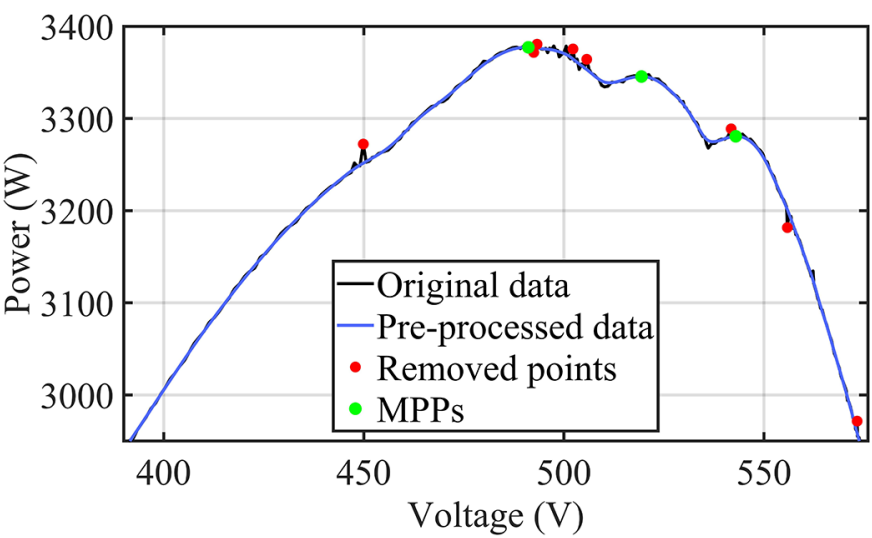

Fig. 3. Example of an original measured and pre-processed $P-U$ curve of the series connection of Strings 1 and 4 near the GMPP.

Uniform irradiance needed to produce that power is close to the highest measured average irradiances of the strings. MPPT of the strings was assumed to work ideally, meaning that the string is operating at its GMPP (or CMPP) unless it is in power limiting mode. If the power at the GMPP (or the CMPP) exceeds the inverter nominal power, the string operates on the high voltage side of the GMPP (or the CMPP) at the lowest voltage where the inverter nominal power is not exceeded.

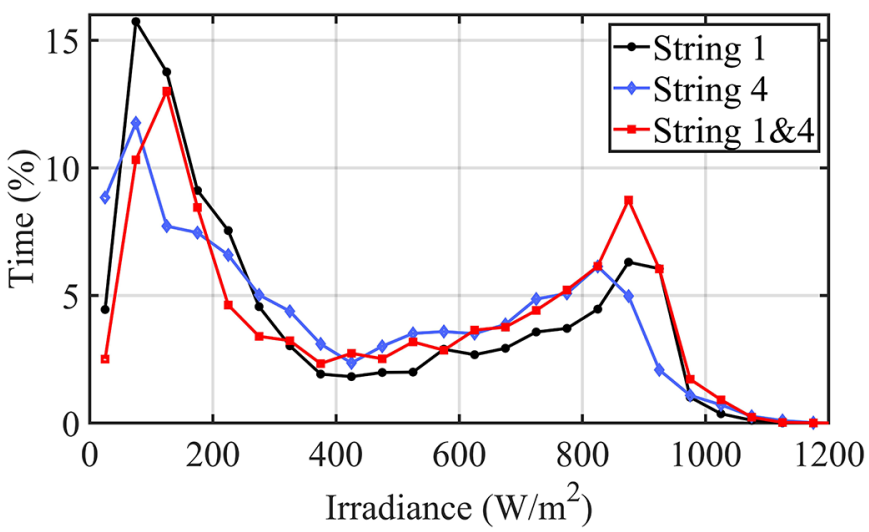

Fig. 4. Distributions of the average irradiances of the studied PV strings calculated as the average of the irradiance measurements of the strings.

\section{Results}

The distributions of the measured voltages of the GMPP and the MPP closest to the nominal MPP voltage are presented in Figure 5 for the studied PV strings. For Strings 4 and $1 \& 4$, both voltages were most of the time below the nominal STC value. This results from the typical operating conditions of the studied PV strings: during most 
Table 3. Shares (\%) of time when the studied PV strings were partially shaded so that the difference between the lowest and highest irradiance measurement of the string exceeded 50, 100 and $200 \mathrm{~W} / \mathrm{m}^{2}$.

\begin{tabular}{llll}
\hline String & $50 \mathrm{~W} / \mathrm{m}^{2}$ & $100 \mathrm{~W} / \mathrm{m}^{2}$ & $200 \mathrm{~W} / \mathrm{m}^{2}$ \\
\hline 1 & 46.6 & 12.2 & 1.5 \\
4 & 1.9 & 0.6 & 0.1 \\
$1 \& 4$ & 62.7 & 28.1 & 1.8 \\
\hline
\end{tabular}

of the studied periods, irradiance was lower and cell temperature was higher than in standard test conditions, and thus the MPP voltages were typically lower than the nominal MPP voltage. Voltage values higher than the nominal MPP voltage were more common for String 1 since it had typically colder operating conditions than the other strings. The effect of operating all the time at the CMPP instead of the GMPP is clearly visible in Figure 5. The voltage of the CMPP was more rarely between $85 \%$ and $95 \%$ and more frequently close to the nominal MPP voltage than the GMPP voltage.

The lowest and highest measured voltages of the GMPP are compiled in Table 4 for the studied strings. The GMPP voltage range was narrower with a higher minimum value and a lower maximum value for String 4 than for Strings 1 and $1 \& 4$, because the length of String 4 is much shorter than the lengths the other two strings. Indeed, the GMPP voltage ranges of String 1 and String $1 \& 4$ were very wide from below $40 \%$ to over $110 \%$ with respect to $U_{\text {MPP sTC }}$ while the GMPP voltage range of String 4 was from $46 \%$ to $104 \%$. The highest measured GMPP voltages for Strings 1 and $1 \& 4$ were over $110 \%$ with respect to $U_{\mathrm{MPP} \text {, STC }}$ and almost $90 \%$ with respect to $U_{\mathrm{OC}}$ sTC. These values are in line with the simulation results of $[18,22]$ where the highest GMPP voltage of a PV string was found to be less than $90 \%$ of $U_{\mathrm{OC} \text {, STC }}$ of the string. Moreover, the measured voltage ranges of the GMPP are in good accord with the experimental results of $[9,23]$ and with the simulation results of [20].

Table 5 presents the lowest and highest measured CMPP voltages for the studied strings. The operational voltage range of a PV inverter can be significantly reduced by operating at the CMPP instead of the GMPP as can be seen by comparing Tables 4 and 5 . The minima of the CMPP voltage ranges were close to $80 \%$ with respect to $U_{\mathrm{MPP}}$, STC for all the studied strings, which is approximately double the GMPP minimum voltage values. However, the highest CMPP voltages were only somewhat larger than the GMPP maximum values. The highest measured CMPP voltages for Strings 1 and $1 \& 4$ were a bit over $90 \%$ with respect to $U_{\mathrm{OC}}$, sTC. The much smaller range of CMPP voltages is an important finding showing that it would be beneficial for PV systems to operate at the CMPP instead of the GMPP. The measured CMPP voltage ranges of Table 5 are largely in line with the results presented in [9]. However, the highest measured CMPP voltages are slightly higher than reported in [9] which is reasonable since only the partially shaded time of PV strings was considered in [9].

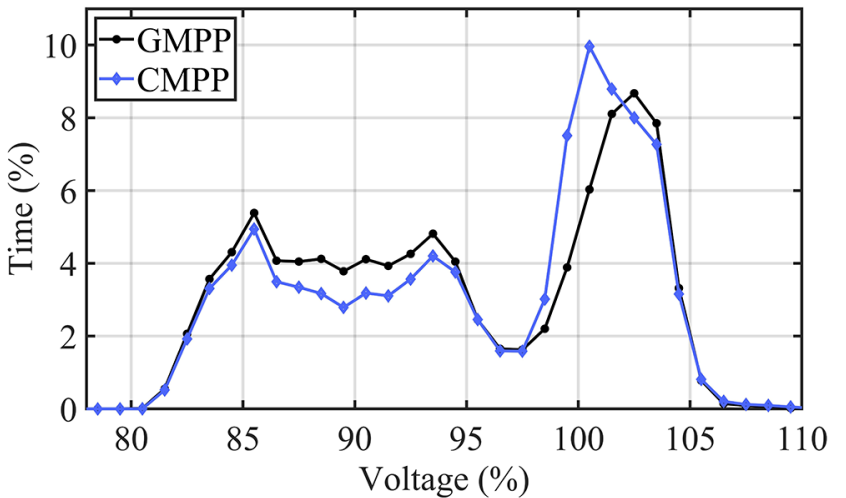

(a)

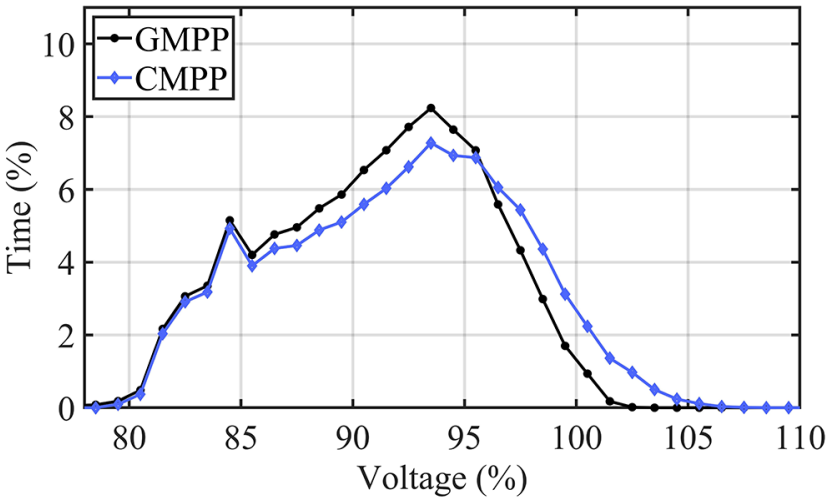

(b)

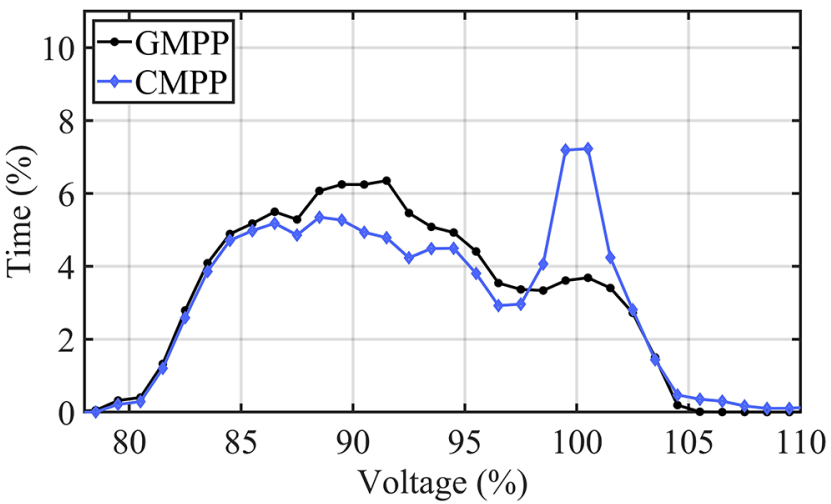

(c)

Fig. 5. Distributions of the measured voltages of the GMPP and CMPP for Strings 1 (a), 4 (b) and $1 \& 4$ (c). The voltages are with respect to the nominal STC MPP voltages. 
Table 4. Measured GMPP voltage ranges for the studied PV strings. The voltage values are with respect to the STC MPP and OC voltages presented in Table 2.

\begin{tabular}{|c|c|c|c|c|}
\hline String & $\begin{array}{l}\text { Minimum voltage } \\
\text { with respect to } \\
U_{\text {MPP, sTC }}(\%) \\
\end{array}$ & $\begin{array}{l}\text { Minimum voltage } \\
\text { with respect to } \\
U_{\mathrm{OC}, \text { sTC }}(\%)\end{array}$ & $\begin{array}{l}\text { Maximum voltage } \\
\text { with respect to } \\
U_{\text {MPP, STC }}(\%)\end{array}$ & $\begin{array}{l}\text { Maximum voltage } \\
\text { with respect to } \\
U_{\text {OC, }} \text { sTC }(\%) \\
\end{array}$ \\
\hline 1 & 38.2 & 29.9 & 113.4 & 88.7 \\
\hline 4 & 45.7 & 35.7 & 104.3 & 81.5 \\
\hline $1 \& 4$ & 37.3 & 29.2 & 111.1 & 86.9 \\
\hline
\end{tabular}

Table 5. Measured CMPP voltage ranges for the studied PV strings. The voltage values are with respect to the STC MPP and OC voltages presented in Table 2.

\begin{tabular}{lllll}
\hline String & $\begin{array}{l}\text { Minimum voltage } \\
\text { with respect to }\end{array}$ & $\begin{array}{l}\text { Minimum voltage } \\
\text { with respect to }\end{array}$ & $\begin{array}{l}\text { Maximum voltage } \\
\text { with respect to }\end{array}$ & $\begin{array}{l}\text { Maximum voltage } \\
\text { with respect to }\end{array}$ \\
& $U_{\mathrm{MPP}, \mathrm{STC}}(\%)$ & $U_{\mathrm{OC}, \mathrm{STC}}(\%)$ & $U_{\mathrm{MPP}, \mathrm{STC}}(\%)$ & $U_{\mathrm{OC}, \mathrm{STC}}(\%)$ \\
\hline 1 & 81.0 & 63.3 & 115.5 & 90.3 \\
4 & 79.2 & 61.9 & 109.3 & 85.5 \\
$1 \& 4$ & 78.3 & 61.2 & 115.4 & 90.2 \\
\hline
\end{tabular}

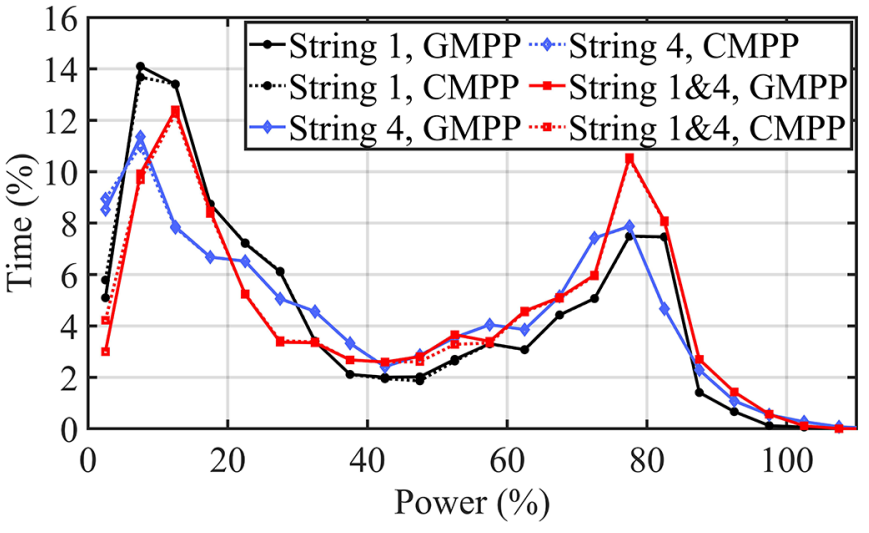

Fig. 6. Distributions of the measured powers of the GMPP and CMPP for the studied PV strings. The powers are with respect to the nominal STC MPP powers.

Figure 6 shows the distributions of the measured powers of the GMPP and CMPP for the studied PV strings. The shapes of the distributions are roughly similar with the irradiance distributions of Figure 4 as expected. By comparing Figures 5 and 6, it can be seen that the differences between the two MPPs in power are much smaller than in voltage. The GMPP and CMPP power distributions of each string are practically overlapping. This results from the typical shape of $P-U$ curves in case of multiple MPPs [9]. There can be large voltage differences between the MPPs while the differences in power remain negligible. For example, in Figure 3, powers of the three MPPs are within 3\% from each other while the voltage difference between the outermost MPPs is $10 \%$. Largest measured power differences between the GMPP and CMPP exist below $15 \%$ and around $50 \%$ of $P_{\mathrm{MPP}}$, sTC. Small differences in power mean that operation at the CMPP instead of the GMPP does not cause significant energy losses.
The maximum clear sky irradiance on the modules of our PV power research plant is just above $900 \mathrm{~W} / \mathrm{m}^{2}$. Therefore, power values higher than $90 \%$ in Figure 6 exceed the expected maximum power of the plant under clear sky irradiance conditions. However, MPP powers higher than $90 \%$ were measured for all strings. The highest measured GMPP powers were $130 \%, 113 \%$ and $107 \%$ with respect to $P_{\mathrm{MPP}}$, stC for Strings 1, 4 and 1\&4, respectively. At these moments, the GMPP was also the CMPP, so these values are also the highest measured CMPP powers. Reason for these cases is the enhanced irradiance caused by clouds, i.e., the cloud enhancement effect. Power values higher than the nominal power were most common for String 4 as it is the physically shortest of the studied strings and thus exposed to strongly enhanced irradiances more easily.

In Figures 5 and 6, the differences between the MPPs were larger for Strings 1 and $1 \& 4$ than for String 4 . Differences in length between the studied strings are the main reason for this. Strings 1 and $1 \& 4$ are physically much longer than String 4. Thus, there are typically larger irradiance and temperature differences between the modules of these strings than between the modules of String 4. Consequently, multiple MPPs exist more often for Strings 1 and $1 \& 4$ than for String 4 . Strings 1 and $1 \& 4$ had more than one MPP around $30 \%$ of the time while String 4 had multiple MPPs only $12 \%$ of the time.

Scatter plot between the measured GMPP power and voltage for String $1 \& 4$ is shown in Figure 7a. The range of the GMPP voltage was quite constant as a function of power: GMPP voltage was most of the time between $80 \%$ and $100 \%$ with respect to $U_{\mathrm{MPP}}$, STC, regardless of the GMPP power. However, there were some cases where the GMPP voltage was below $80 \%$ or clearly above $100 \%$. In those cases, the GMPP power was typically from $20 \%$ to $60 \%$ with respect to $P_{\mathrm{MPP}}$. sTC. Figure $7 \mathrm{~b}$ shows similar scatter plot between the CMPP power and voltage. The pattern is quite similar than for the GMPP in Figure 7a. 


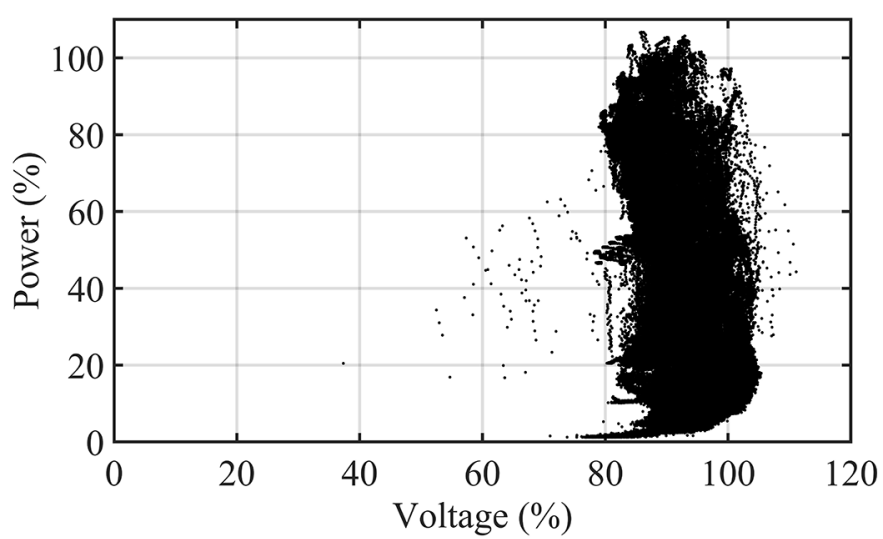

(a)

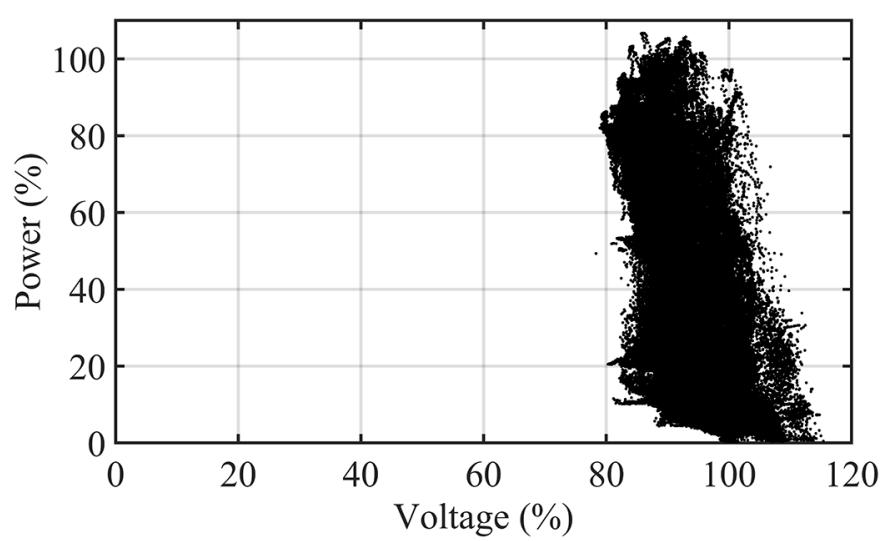

(b)

Fig. 7. Scatter plots between the measured power and voltage at the GMPP (a) and CMPP (b) for String $1 \& 4$. The values are with respect to the nominal STC MPP values.

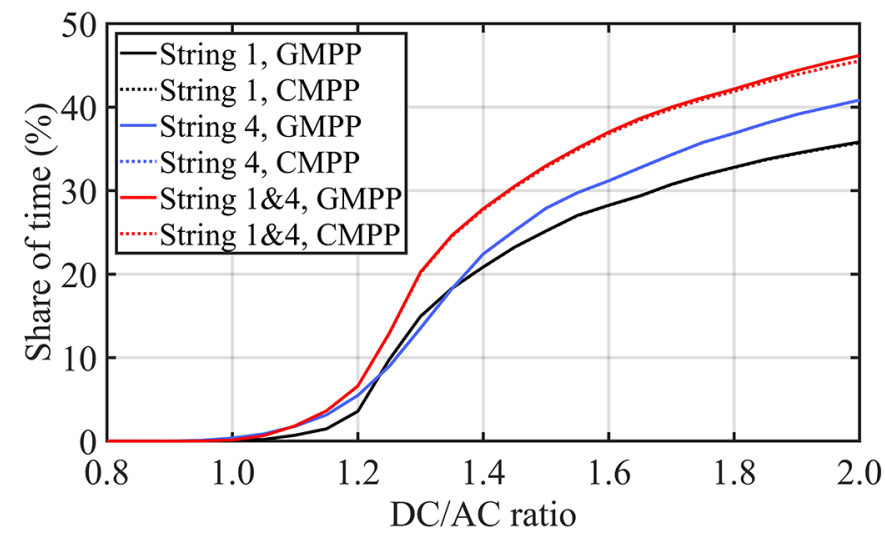

Fig. 8. Shares of time when the studied PV strings were in power limiting mode when operating at the GMPP or at the CMPP as a function of the $\mathrm{DC} / \mathrm{AC}$ ratio.

However, there are two clear differences. Firstly, voltage values lower than $78.3 \%$ did not exist for the CMPP. Secondly, voltage values higher than $105 \%$ were much more common for the CMPP than for the GMPP. These high voltage values typically occurred when the CMPP power was less than $40 \%$ with respect to $P_{\mathrm{MPP}}$, sTC.

Figure 8 presents the shares of time when the studied $\mathrm{PV}$ strings operated in power limiting mode as a function of $\mathrm{DC} / \mathrm{AC}$ ratio. Naturally, the shares of time when the $\mathrm{PV}$ strings operated in power limiting mode increased with the increasing $\mathrm{DC} / \mathrm{AC}$ ratio, since the irradiance level needed to produce $\mathrm{PV}$ power exceeding the nominal inverter power decreases as the DC/AC ratio increases. With small DC/ $\mathrm{AC}$ ratios below 1.1, all the strings spent only negligible time in power limiting mode, since the maximum clear sky irradiance at Tampere's latitude is just above $900 \mathrm{~W} / \mathrm{m}^{2}$. The share of time when the studied strings were in power limiting mode increased rabidly with the increasing DC/ $\mathrm{AC}$ ratio after value 1.2 and all the strings were more than one third of the time in power limiting mode when the DC/ $\mathrm{AC}$ ratio approached 2.0. The differences between the GMPP and CMPP were very small but the shares were a bit larger for the GMPP.
Figure 9 shows the highest, median and lowest operating voltage as a function of the $\mathrm{DC} / \mathrm{AC}$ ratio for the studied strings while operating at the GMPP or at the CMPP. The maximum and median operating voltages increased slightly with the increasing $\mathrm{DC} / \mathrm{AC}$ ratio for all the strings since the strings were operating on the high voltage side of the GMPP (or the CMPP) in power limiting mode. The maximum GMPP voltage approached the maximum CMPP voltage and the difference in voltage ranges between the MPPs increased as the $\mathrm{DC} / \mathrm{AC}$ ratio increased. The maximum GMPP voltage of Strings 1, 4 and $1 \& 4$ reached the maximum CMPP voltage with $\mathrm{DC} / \mathrm{AC}$ ratios of $1.0,1.25$ and 1.35 , respectively. The median GMPP and CMPP voltages overlapped for all DC/AC ratios and the highest GMPP and CMPP voltages overlapped for most $\mathrm{DC} / \mathrm{AC}$ ratios. On the contrary, the lowest GMPP and CMPP voltage values stayed almost constant as a function of the $\mathrm{DC} / \mathrm{AC}$ ratio for all the strings having a major difference between them, as earlier noticed. This indicates that the voltage of the MPP closest to the nominal MPP voltage could be used instead of the GMPP voltage also when applying power curtailment.

Narrowing of the operating voltage range when operating at the CMPP instead of the GMPP is further illustrated in Figure 10 where the differences in voltage ranges between the MPPs are presented as a function of the $\mathrm{DC} / \mathrm{AC}$ ratio. At small $\mathrm{DC} / \mathrm{AC}$ ratios, the differences in voltage ranges between the MPPs increased as the $\mathrm{DC} / \mathrm{AC}$ ratio increased and were constant with a $\mathrm{DC} / \mathrm{AC}$ ratio range from 1.4 to 2.0 . On that range, the CMPP voltage range of String 4 was $35 \%$ narrower than the GMPP voltage range with respect to $U_{\mathrm{MPP}}$, STC. For the longer strings the corresponding values were over $40 \%$. The differences between the studied strings decreased with the increasing $\mathrm{DC} / \mathrm{AC}$ ratio, on average.

The relative energy losses due to operation at the CMPP instead of the GMPP are presented in Figure 11 as a function of the $\mathrm{DC} / \mathrm{AC}$ ratio. The relative energy losses due to operation at the CMPP increased with increasing string length. Moreover, the relative energy losses increased with the increasing $\mathrm{DC} / \mathrm{AC}$ ratio for Strings 1 and $1 \& 4$ while they decreased a bit for String 4. However, only negligible 


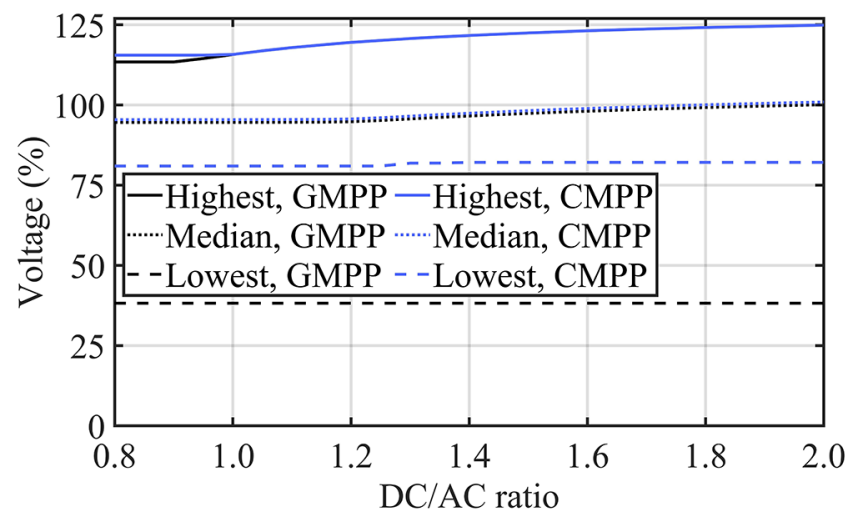

(a)

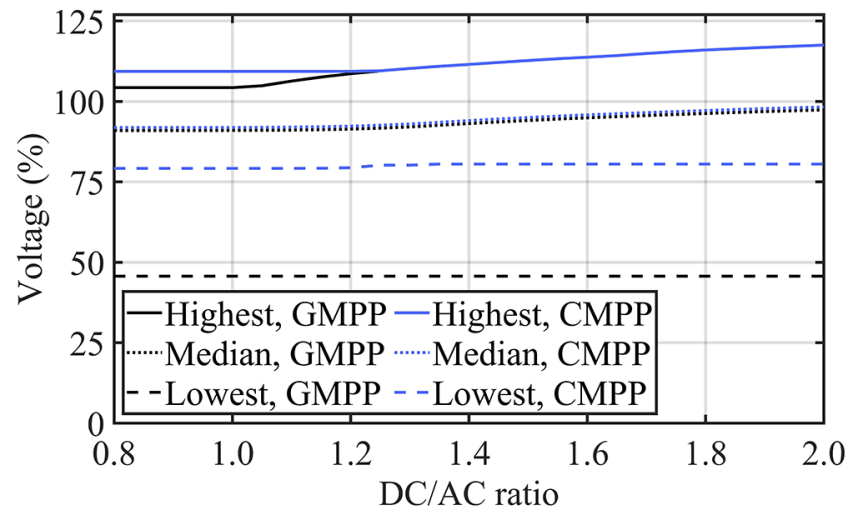

(b)

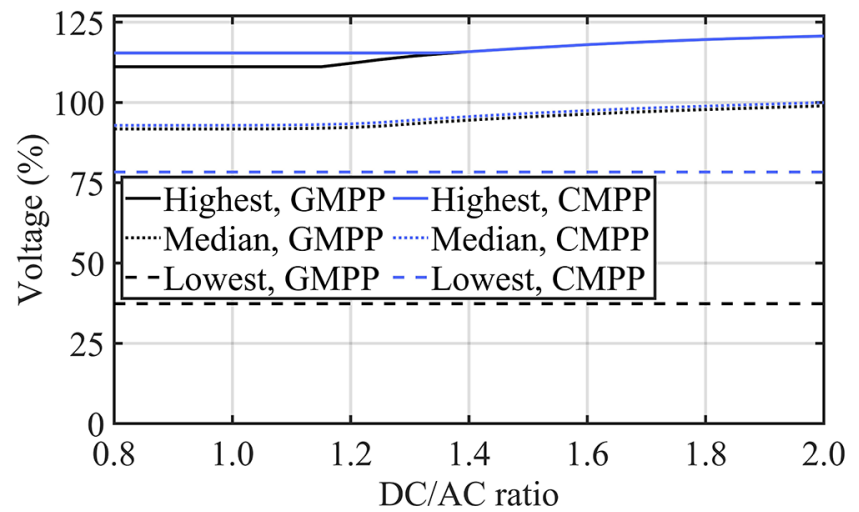

(c)

Fig. 9. Highest, median and lowest operating voltage as a function of the DC/AC ratio while operating at the GMPP or at the CMPP for Strings 1 (a), 4 (b) and 1\&4 (c). The voltages are with respect to the nominal STC MPP voltages.

amount of energy would be lost if the PV strings operated all the time at the CMPP instead of the GMPP. This demonstrates that the wide operating voltage range when the GMPP is followed can be significantly reduced by operating at the MPP closest to the nominal MPP voltage at a cost of only marginal energy losses.

Figure 12 presents the relative energy losses due to power curtailment as a function of the $\mathrm{DC} / \mathrm{AC}$ ratio. Differences in energy losses between the strings were quite small. The relative energy losses due to power curtailment were much larger than the relative energy losses due to operation at the CMPP instead of the GMPP. For

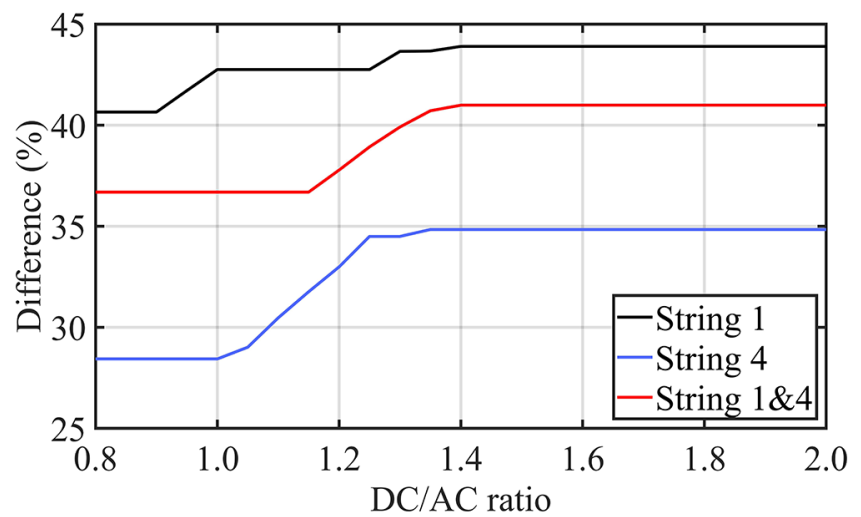

Fig. 10. Differences in operating voltage ranges between the GMPP and CMPP as a function of the DC/AC ratio calculated with respect to the nominal STC MPP voltages.

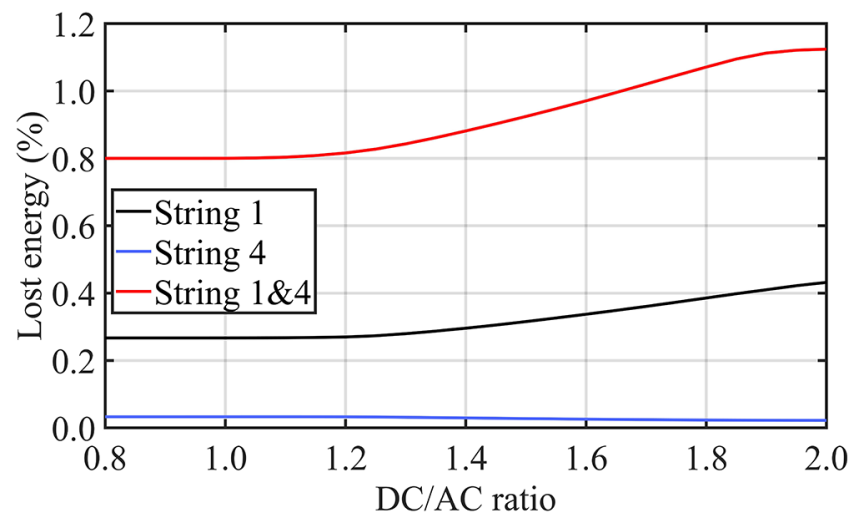

Fig. 11. Relative energy losses of the studied PV strings due to operation at the CMPP instead of the GMPP as a function of the $\mathrm{DC} / \mathrm{AC}$ ratio calculated with respect to energy produced at the GMPP.

example, with the $\mathrm{DC} / \mathrm{AC}$ ratio of 1.6 , over $14 \%$ of available energy in each string would be lost due to power curtailment. The losses increased strongly with the increasing $\mathrm{DC} / \mathrm{AC}$ ratio being around $30 \%$ with the $\mathrm{DC} / \mathrm{AC}$ ratio of 2.0. With small $\mathrm{DC} / \mathrm{AC}$ ratios below 1.1, the strings were in power limiting mode only during cloud enhancement. As explained earlier, the share of time spent in power limiting mode increases with the increasing DC/ $\mathrm{AC}$ ratio since the irradiance level needed to produce $\mathrm{PV}$ power exceeding the nominal inverter power decreases.

\section{Discussion}

Differences in operating conditions of the studied strings might have distorted the comparison between the strings. The $I-U$ curve measurements of the strings were performed one at a time on different days. Naturally, it would have been ideal to measure all the strings simultaneously. Unfortunately, that option was not available. However, most of the measurement days were close to each other since all the measurements were done during two periods 


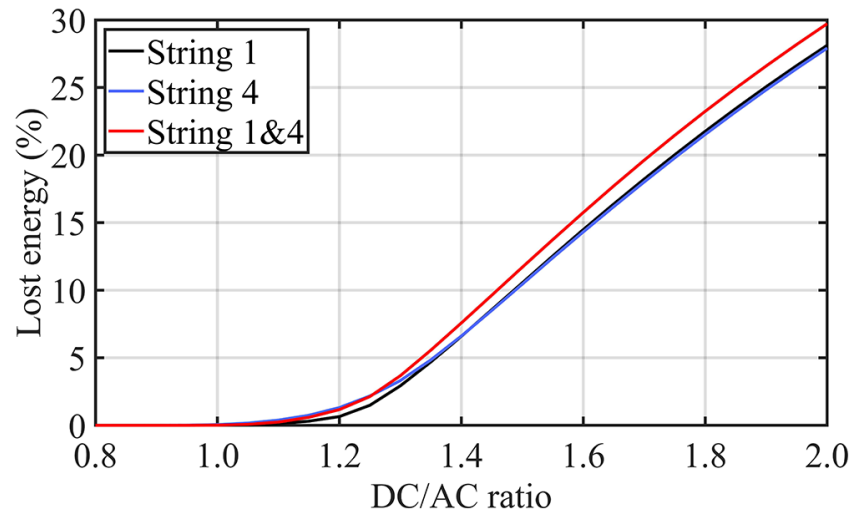

Fig. 12. Relative energy losses of the studied PV strings due to power curtailment as a function of the $\mathrm{DC} / \mathrm{AC}$ ratio calculated with respect to energy produced at the GMPP without power curtailment.

from 7 August to 19 August 2020 and from 23 April to 8 June 2021. In addition to measurement periods, also shadings by nearby built structures caused occasionally differences in operating conditions of the strings. However, the studied strings are installed very close by each other. Thus, shadings by nearby built structures did not cause major differences between the strings. As the average irradiance distributions in Figure 4 show, there were no major differences in irradiance condition between the studied strings. However, there were some differences on the measured average back-sheet temperatures of PV modules between the strings. The average temperatures for Strings 4 and $1 \& 4$ were close to each other: 34.7 and $35.2^{\circ} \mathrm{C}$, respectively. However, the average temperature for String 1 was lower $27.0^{\circ} \mathrm{C}$. The lower operating temperature results in higher operating and MPP voltages and thus affects mainly the results of String 1 in Figures 5 and 9 .

Naturally, the measurement period affects the obtained results. Somewhat higher voltage and power values would have been obtained if the measurements were performed on mid-summer with higher irradiance levels. Moreover, the location where the measurements were performed affects the obtained results. Thus, the exact values are representative of only high-latitude locations. However, the general observations of the studied phenomena and the conclusions drawn from the results are not regionally bounded but can be applied globally.

The results indicate that a PV inverter should be sized based on the expected maximum clear-sky irradiance if the intention is to minimise energy losses due to power curtailment. In the Tampere region, the theoretical peak irradiance value on a clear sky day during the studied period is approximately $900 \mathrm{~W} / \mathrm{m}^{2}$ meaning that with a $\mathrm{DC} / \mathrm{AC}$ ratio of around 1.1 the $\mathrm{PV}$ strings are in power limiting mode only during cloud enhancement events. Figure 12 shows that, with a DC/AC ratio of 1.1, energy losses due to power curtailment were from $0.12 \%$ to $0.38 \%$, increasing strongly with higher DC/AC ratios. Moreover, the relative energy losses for Strings 1 and $1 \& 4$ due to operation at the CMPP instead of the GMPP (Fig. 11) were quite constant at $\mathrm{DC} / \mathrm{AC}$ ratios lower than 1.1 increasing with higher DC/AC ratios. Use of energy storage systems would decrease energy losses caused by power curtailment. Use of energy storage systems was out of the scope of this study but sizing and operation of energy storage systems used in PV power plants are interesting topics for future studies.

\section{Conclusions}

In this article, an experimental study of the GMPP characteristics of PV strings and of a scenario in which the MPP closest to the nominal MPP voltage is always the operating point instead of the GMPP was presented. In total, 1,296,000 $I-U$ curves of $3 \mathrm{PV}$ strings measured over $360 \mathrm{~h}$ were analysed. Moreover, the effects of inverter sizing on the operating point behaviour of the strings were studied by altering the DC/AC ratio from 0.8 to 2.0. Such an exhaustive study based on actual electrical measurements of the optimal operating point of $\mathrm{PV}$ generators, also considering the effects of inverter sizing on the selection of the operating point, has not been presented earlier.

The experimental results demonstrate that it would be beneficial for PV systems to operate at the MPP closest to the nominal MPP voltage instead of the GMPP. For the studied strings of 6,17 and 23 series-connected PV modules, a very wide GMPP voltage range from around $40 \%$ to around $110 \%$, with respect to the nominal MPP voltage, can be significantly reduced by operating at the CMPP with a voltage range from around $80 \%$ to around $115 \%$. Moreover, the results show that only negligible amount of energy would be lost if the PV strings operated all the time at the CMPP instead of the GMPP. Thus, the wide operating voltage range when the GMPP is followed can be significantly reduced by operating at the MPP closest to the nominal MPP voltage at a cost of only negligible energy losses. The experimental results presented in this article confirm the findings of earlier simulation studies.

Energy losses due to power curtailment were found to be much larger than energy losses due to operation at the CMPP instead of the GMPP. For example, with a typical $\mathrm{DC} / \mathrm{AC}$ ratio of 1.6 , over $14 \%$ of available energy would be lost due to power curtailment. The losses increased strongly with the increasing $\mathrm{DC} / \mathrm{AC}$ ratio after value 1.2 being close to $30 \%$ with the $\mathrm{DC} / \mathrm{AC}$ ratio of 2.0 .

The results indicate that, if the intention is to minimise energy losses, the inverter should be sized based on the expected maximum clear-sky irradiance so that the PV strings would be in power limiting mode only during cloud enhancement. In the Tampere region, that means a DC/ $\mathrm{AC}$ ratio of around 1.1. With this $\mathrm{DC} / \mathrm{AC}$ ratio, energy losses due to power curtailment were from $0.12 \%$ to $0.38 \%$, increasing strongly with higher DC/AC ratios. Moreover, the relative energy losses for the strings of 17 and 23 modules due to operation at the CMPP instead of the GMPP were quite constant at DC/AC ratios below 1.1 and started to increase after that. Sizing of energy storage systems used in PV power plants would be an interesting and fruitful topic for continuation of this study. 
We acknowledge the financial support from Business Finland for the research reported in this article.

\section{Author contribution statement}

Kari Lappalainen: Conceptualization, Methodology, Formal analysis, Investigation, Writing (Original Draft). Seppo Valkealahti: Conceptualization, Writing (Review \& Editing).

\section{References}

1. A. Cabrera-Tobar, E. Bullich-Massagué, M. AragüésPeñalba, O. Gomis-Bellmunt, Review of advanced grid requirements for the integration of large scale photovoltaic power plants in the transmission system, Renew. Sustain. Energy Rev. 62, 976 (2016)

2. S. Shivashankar, S. Mekhilef, H. Mokhlis, M. Karimi, Mitigating methods of power fluctuation of photovoltaic (PV) sources - A review, Renew. Sustain. Energy Rev. 59, $1170(2016)$

3. X. Chen, Y. Du, H. Wen, L. Jiang, W. Xiao, ForecastingBased Power Ramp-Rate Control Strategies for Utility-Scale PV Systems, IEEE Trans. Industrial Electronics 66, 1862 (2019)

4. T.S. Wurster, M.B. Schubert, Mismatch loss in photovoltaic systems, Sol. Energy 105, 505 (2014)

5. K. Lappalainen, S. Valkealahti, Effects of PV array layout, electrical configuration and geographic orientation on mismatch losses caused by moving clouds, Sol. Energy 144, 548 (2017)

6. C.G. Lee, W.G. Shin, J.R. Lim, G.H. Kang, Y.C. Ju, H.M. Hwang, H.S. Chang, S.W. Ko, Analysis of electrical and thermal characteristics of PV array under mismatching conditions caused by partial shading and short circuit failure of bypass diodes, Energy 218, 119480 (2021)

7. B. Yang, T. Zhu, J. Wang, H. Shu, T. Yu, X. Zhang, W. Yao, L. Sun, Comprehensive overview of maximum power point tracking algorithms of PV systems under partial shading condition, J. Clean. Prod. 268, 121983 (2020)

8. A.F. Mirza, M. Mansoor, K. Zhan, Q. Ling, High-efficiency swarm intelligent maximum power point tracking control techniques for varying temperature and irradiance, Energy 228, 120602 (2021)

9. K. Lappalainen, S. Valkealahti, Experimental study of the maximum power point characteristics of partially shaded photovoltaic strings, Appl. Energy 301, 117436 (2021)

10. H.X. Wang, M.A. Munoz-García, G.P. Moreda, M.C. Alonso-García, Optimum inverter sizing of grid-connected photovoltaic systems based on energetic and economic considerations, Renew. Energy 118, 709 (2018)

11. J.M.S. Callegari, A.F. Cupertino, V.N. Ferreira, E.M.S. Brito, V.F. Mendes, H.A. Pereira, Adaptive dc-link voltage control strategy to increase PV inverter lifetime, Microelectron. Reliab. 100-101, 113439 (2019)

12. G.A. Rampinelli, A. Krenzinger, F. Chenlo Romero, Mathematical models for efficiency of inverters used in grid connected photovoltaic systems, Renew. Sustain. Energy Rev. 34, 578 (2014)

13. J.D. Mondol, Y.G. Yohanis, B. Norton, Optimal sizing of array and inverter for grid-connected photovoltaic systems, Sol. Energy 80, 1517 (2006)

14. J. Zhu, R. Bründlinger, T. Mühlberger, T.R. Betts, R. Gottschalg, Optimised inverter sizing for photovoltaic systems in high-latitude maritime climates, IET Renewable Power Generation 5, 58 (2011)

15. K. Lappalainen, J. Kleissl, Analysis of the cloud enhancement phenomenon and its effects on photovoltaic generators based on cloud speed sensor measurements, J. Renew. Sustain. Energy 12, 043502 (2020)

16. J. Luoma, J. Kleissl, K. Murray, Optimal inverter sizing considering cloud enhancement, Sol. Energy 86, 421 (2012)

17. K. Peippo, P.D. Lund, Optimal sizing of solar array and inverter in grid-connected photovoltaic systems, Sol. Energy Mater. Sol. Cells 32, 95 (1994)

18. A.M.S. Furtado, F. Bradaschia, M.E. Cavalcanti, L.R. Limongi, A Reduced Voltage Range Global Maximum Power Point Tracking Algorithm for Photovoltaic Systems Under Partial Shading Conditions, IEEE Trans. Industrial Electronics 65, 3252 (2018)

19. K. Lappalainen, S. Valkealahti, Number of maximum power points in photovoltaic arrays during partial shading events by clouds, Renew. Energy 152, 812 (2020)

20. K. Lappalainen, S. Valkealahti, Fluctuation of PV array global maximum power point voltage during irradiance transitions caused by clouds, IET Renewable Power Generation 13, 2864 (2019)

21. K. Ding, X. Wang, Q.X. Zhai, J.W. Xu, J.W. Zhang, H.H. Liu, Improved global maximum power point tracking method based on voltage interval for PV array under partially shaded conditions, J. Power Electron. 14, 722 (2014)

22. M. Boztepe, F. Guinjoan, G. Velasco-Quesada, S. Silvestre, A. Chouder, E. Karatepe, Global MPPT Scheme for Photovoltaic String Inverters Based on Restricted Voltage Window Search Algorithm, IEEE Trans. Industrial Electronics 61, 3302 (2014)

23. K. Lappalainen, S. Valkealahti, Experimental Study of the Behaviour of the Global MPP of Partially Shaded PV Strings, in Proceedings of 37th European Photovoltaic Solar Energy Conference, Vol. I, 1501 (2020). https://doi.org/ 10.4229/EUPVSEC20202020-5CV.3.4.

24. D. Torres Lobera, A. Mäki, J. Huusari, K. Lappalainen, T. Suntio, S. Valkealahti, Operation of TUT Solar PV Power Station Research Plant under Partial Shading Caused by Snow and Buildings, Int. J. Photoenergy 2013, 837310 (2013)

25. K. Lappalainen, S. Valkealahti, Analysis of shading periods caused by moving clouds, Sol. Energy 135, 188 (2016)

Cite this article as: Kari Lappalainen, Seppo Valkealahti, Analysis of the operation of PV strings at the MPP closest to the nominal MPP voltage instead of the global MPP based on measured current-voltage curves, EPJ Photovoltaics 13, 4 (2022) 